\title{
ON QUADRATIC, HERMITIAN AND BILINEAR FORMS*
}

\author{
BY \\ LEONARD EUGENE DICKSON
}

Introduction.

Part $I$ is concerned with the reduction of quadratic forms in an arbitrary field to canonical types, a problem hitherto treated only for finite fields and for the field of all real or all complex numbers.

Part II treats of the reduction of hermitian forms in a field $Q$ obtained by the adjunction to an arbitrary field $F$ of a root of a quadratic equation belonging to and irreducible in $F$. The problem is completely solved when $F$ is any finite field, the field of all real numbers, or the field of all rational numbers.

Part III deals with the bilinear forms in an arbitrary field $F$ which are invariant under a given substitution $S$ with coefficients in $F$. The necessary and sufficient conditions on $S$ for the existence of such invariants are obtained, and the reduction of the invariants to a single normal form is effected by a transformation commutative with $S$. Here and in Part IV use is made of the writer's determination of the canonical form of a linear transformation in an arbitrary field. $\dagger$

Part IV treats the analogous questions on quadratic forms and gives the generalization to an arbitrary field $F$ of JoRDAN's recent results for the case of a field of order $p$, a prime. $\ddagger$ The explicit form of the general invariant is determined with the same ease, but the difficult problem of its reduction to canonical forms by substitutions commutative with $S$ becomes much more troublesome for an arbitrary field than for a finite field. When $F$ does not have modulus 2 , the general character of the result may be indicated as follows. As seminormal forms of the invariant we obtain

$$
\sum B_{i}+\sum a_{i} H_{i}+\sum a_{i}^{\prime} H_{i}^{\prime}+\cdots+\sum A_{i}+\sum b_{i} Q_{i}+\sum b_{i}^{\prime} Q_{i}^{\prime}+\cdots,
$$

where the invariants $B_{i}, H_{i}, A_{i}$ and $Q_{i}$ have respectively the character of bilinear, hermitian, alternate bilinear and quadratic forms, each with completely

\footnotetext{
* Presented to the Society (Chicago) December 30, 1905. Received for publication November 26,1905 .

† American Journal of Mathematics, vol. 24 (1902), pp. 101-108.

$\ddagger$ Mémoire sur les formes quadratiques, suivant un module premier $p$, invariantes par une substitution linéaire donnée, Journal de Mathématiques, ser. 6, vol. 1 (1905), pp. 217-284.
} 
fixed coefficients, while $b_{i}, b_{i}^{\prime}, \cdots$ are any non-vanishing elements of $F$ and $a_{i}, a_{i}^{\prime}, \ldots$, any non-vanishing elements of certain fields $F(\rho)$. The question of ultimate normal forms is the question of the extent to which these parameters can be specialized by the application of a substitution commutative with $S$. It is shown that normalization must take place in each sum separately, that the normalization of the quadratic function $\sum b_{a} Q_{a}$ of certain variables $x_{j}^{a}$ must be made by a substitution $T$ on the $x_{0}^{a}$, the same substitution on the $x_{i}^{a}$, the same on $x_{2}^{a}$, etc., with analogous remarks on the sums $\sum a_{i} H_{i}, \ldots$ But the effect on the $b_{i}$ is the same as if we had applied to $\sum b_{a}\left(x_{0}^{a}\right)^{2}$ the substitution $T$ on the variables $x_{0}^{a}$ alone. Moreover, this partial substitution on the $x_{0}^{a}$ may be chosen arbitrarily. Hence the problem of normalizing $\sum_{a=1}^{l} b_{a} Q_{a}$ by a substitution commutative with $S$ and cogredient in the various sets of variables is replaced by the problem (treated in Part I) of normalizing an $l$-ary quadratic form in $F$ by means of unrestricted $l$-ary substitutions in $F$. Similarly, the problem on $\sum a_{i} H_{i}$ reduces to that on hermitian forms (Part II).

\section{Reduction of quadratic forms* in a general field $F$.}

1. Within any field $F$, not having modulus 2 , a quadratic form of non-vanishing determinant is linearly reducible to $\dagger$

$$
q \equiv \sum_{i=1}^{n} a_{i} x_{i}^{2} \quad\left(\text { each } a_{i} \text { an element } \neq 0 \text { of } F\right) .
$$

Hence for the field of real numbers the canonical types are

$$
f_{.} \equiv \sum_{i=1}^{\infty} x_{i}^{2}-\sum_{i=\alpha+1}^{n} x_{i}^{2} .
$$

For $s \neq \sigma, f_{c}$ cannot be transformed into $f_{\sigma}$ by a real $n$-ary linear substitution; this invariance of $s$ is the JACOBI-SYLvESTER law of inertia of real quadratic forms. $\ddagger$

2. Under the transformation

$q$ becomes

$$
x_{i}=\sum_{j=1}^{n} b_{i j} y_{j} \quad(i=1, \cdots, n),
$$

$$
\sum_{j=1}^{n} A_{j} y_{j}^{2}+2 \sum_{j, k, j<k}^{1, \ldots n} B_{j k} y_{j} y_{k}, \quad A_{j} \equiv \sum_{i=1}^{n} a_{i} b_{i j}^{2}, \quad B_{j k} \equiv \sum_{i=1}^{n} a_{i} b_{i j} b_{i k} .
$$

We discuss the question: Given $b_{11}, b_{21}, \cdots, b_{n 1}$, in the general field $F$, such that $A_{1} \neq 0$, can we determine elements $b_{i j}(j>1)$ of $F$, such that every

* The same treatment applies to the reduction cf sy mmetric bilinear forms by cogredient transformations of the two sets of variables.

† The usual proof for the field of all real numbers is valid for $F$. Or we may proceed as in $z 6$, identifying every element with its conjugate.

$\ddagger$ References in Baltzer, Determinanten, 5th ed., p. 176. 
$B_{j k}=0$ and $\Delta \equiv\left|b_{i j}\right| \neq 0$ ? Since the $b_{i 1}$ enter the question symmetrically, we may assume that $b_{11} \neq 0$. The conditions $B_{1 k}=0$ are satisfied if we take

$$
b_{1 k}=-a_{1}^{-1} b_{11}^{-1} \sum_{i=2}^{n} a_{i} b_{i 1} b_{i k} \quad(k=2, \cdots, n) .
$$

If, with these values inserted in $\Delta$, we remove the factor $a_{1}^{-1} b_{11}^{-1}$ from the first row of $\Delta$, then multiply the $i$ th row by $a_{i} b_{i 1}$ and add the sum to the first row, for $i=2, \ldots, n$, we find that

$$
\Delta=a_{1}^{-1} b_{11}^{-1} A_{1} \Delta_{11}, \quad \Delta_{11} \equiv\left|b_{i x}\right| \quad(i, s=2, \cdots, n) .
$$

If $n=2$, we take $b_{22} \neq 0$ and obtain an affirmative answer to our question. Let next $n>2$. In $B_{j k}=0(2 \leqq j<k)$, we replace $b_{1 j}$ and $b_{1 k}$ by their values and get $B_{j k}^{\prime} \equiv \sum_{i=2}^{n} R_{i} b_{i j} b_{i k}+\sum_{i, t, i \neq t}^{2, \ldots{ }^{n}} P_{i t} b_{i j} b_{t k}=0, \quad R_{i} \equiv a_{i}^{2} b_{i 1}^{2}+a_{1} a_{i} b_{11}^{2}, \quad P_{i t} \equiv a_{i} a_{1} b_{i 1} b_{t 1}$.

(i) Suppose first that every $R_{i}=0(i=2, \ldots, n)$. Then every $b_{i 1} \neq 0$, $P_{i t} \neq 0$. With the exception of $b_{32}$, we take every $b_{i x}=0(i, s=2, \cdots, n ; i>s)$. We assign any value not zero to $b_{i i}(i>1)$; and any values to $b_{23}, b_{32}$ such that $b_{23} b_{32}=-b_{22} b_{33}$. Then $\Delta_{11}=2 b_{22} b_{33} \ldots b_{n n} \neq 0$. Finally, for $l=4, \ldots, n$, we set

$$
C_{i l} \equiv \sum_{l \neq i}^{l=\cdots, \ldots, l} P_{i t} b_{n}=0 \quad(i=2, \cdots, l-1) .
$$

Then conditions $B_{j k}^{\prime} \doteq 0(j, k=2, \ldots, n ; j<k)$, are all satisfied, since the coefficient of $b_{i j}$ equals $C_{i k}$ if $k \geqq 4$, while for $k=3, j=2$, the condition reduces to $P_{23}\left(b_{22} b_{33}+b_{32} b_{23}\right)=0$. But for a fixed $l, l>3$, equations $C_{i l}=0$ serve to express the $b_{l l}(t=2, \cdots, l-1)$ in terms of $b_{l}$, since the determinant of the coefficients of the former is*

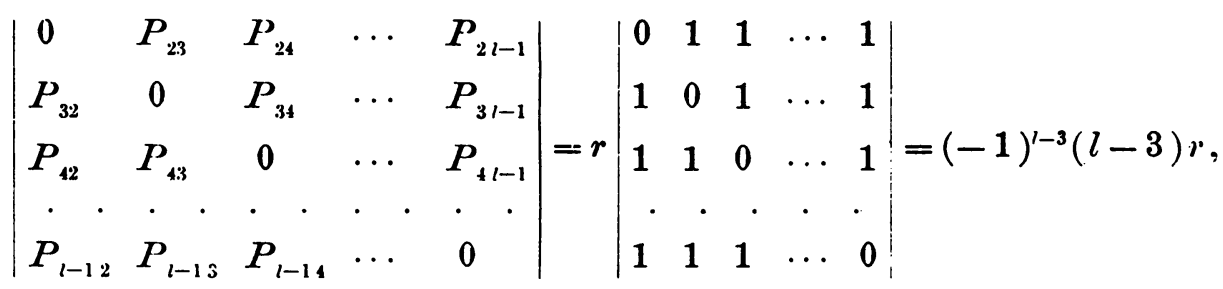

* To reach the second determinant, replace each $P_{i t}$ by $a_{i} a_{t} b_{i 1} b_{t 1}$, remove the factor $a_{i} b_{i 1}$ from the $(i-1)$ th row, and the factor $a_{t} b_{t 1}$ from the $(t-1)$ th colnmn. To evaluate the second determinant, subtract the first row from each of the remaining rows, then add the $2^{d}, \cdots,(l-2)$ th columns to the first. The resulting determinant has the first row $l-3,1,1, \cdots, 1$, and zeros elsewhere outside the main diagonal. 
where $r \equiv a_{2}^{2} a_{3}^{2} \cdots a_{l-1}^{2} b_{21}^{2} b_{31}^{2} \cdots b_{l-11}^{2} \neq 0$. Hence the equations $C_{i l}=0$ can all be satisfied if $* F$ does not have a modulus $\leqq n-3$.

(ii) If not every $R_{i}$ vanishes, we may set $R_{2} \neq 0$ in view of the symmetry. We determine the $b_{2 k}$ to make the coefficient of $b_{2 j}$ in $B_{j k}^{\prime}$ vanish :

$$
b_{2 k}=-R_{2}^{-1} \sum_{t=3}^{n} P_{2 t} b_{t k} \quad(k=3, \cdots, n) .
$$

We give to $b_{22}$ any value $\neq 0$ and set $b_{i 2}=0(i=3, \ldots, n)$. Then $B_{2 k}^{\prime} \equiv 0$ and

$$
\Delta_{11}=b_{22} \Delta_{22}, \quad \Delta_{22} \equiv\left|b_{i s}\right| \quad(i, s=3, \cdots, n) .
$$

If $n=3$, we take $b_{33} \neq 0$ and obtain an affirmative answer to our question. Let next $n>3$. In $B_{j k}^{\prime}=0(3 \leqq j<k)$, we replace $b_{t k}$ for $t=2$ by its value and obtain

$$
B_{j k}^{\prime \prime} \equiv \sum_{i=3}^{n} b_{i j}\left\{R_{i}^{\prime} b_{i k}+\sum_{t \neq i}^{t=3, \ldots, n} P_{i t}^{\prime} b_{t k}\right\}=0, \quad R_{i}^{\prime} \equiv R_{2} R_{i}-P_{2 i}^{2}, \quad P_{i t}^{\prime} \equiv a_{1} a_{2} b_{11}^{2} P_{i t},
$$

the value of $P_{i t}^{\prime}$ being initially $R_{2} P_{i t}-P_{2 i} P_{2 t}$. The present problem - to determine the $b_{i s}(i \geqq 3, s \geqq 3)$ so that their determinant $\Delta_{22} \neq 0$ and each $B_{j k}^{\prime \prime}=0(3 \leqq j<k)$ - is the exact analogue of the former problem - to determine the $b_{i s}(i \geqq 2, s \geqq 2)$ so that $\Delta_{11} \neq 0$ and each $B_{j k}^{\prime}=0(2 \leqq j<k)$. After suitable repetitions of the argument, the final problem is to determine the $b_{i s}(i, s=n-1, n)$ so that $\left|b_{i s}\right| \neq 0$ and

$$
B_{n-1 n}^{*} \equiv \sum_{i=n-1}^{n} b_{i n-1}\left\{R_{i}^{*} b_{i n}+\sum_{t \neq i}^{t=n-1, n} P_{i t}^{*} b_{t n}\right\}=0, \quad P_{n-1 n}^{*}=P_{n n-1}^{*} \equiv P .
$$

If $R_{n-1}^{*}, R_{n}^{*}$ and $P$ all vanish, we may choose the $b$ 's to be any elements of determinant not zero. In the contrary case, we take

$$
b_{n-1 n-1}=R_{n}^{*} b_{n n}+P_{n-1 n}, \quad b_{n n-1}=-R_{n-1}^{*} b_{n-1 n}-P b_{n n} .
$$

Then $B_{n-1 n}^{*} \equiv 0,|b|=R_{n}^{*} b_{n n}^{2}+2 P b_{n n} b_{n-1 n}+R_{n-1}^{*} b_{n-1 n}^{2} \neq 0$.

THEOREM. If $F$ is any field not $\dagger$ having a modulus $\leqq n-3$, there exists an n-ary linear transformation $\left(b_{i j}\right)$ in $F$, with preassigned values of $b_{11}, b_{21}, \ldots, b_{n 1}$ making $\sum_{i=1}^{n} a_{i} b_{i 1}^{2} \neq 0$, which replaces a given quadratic form $\sum_{i=1}^{n} a_{i} x_{i}^{2}$ by one of the type $\sum_{i=1}^{n} A_{i} x_{i}^{2}$ with $A_{1}=\sum_{i=1}^{n} a_{i} b_{i 1}^{2}$.

* This condition is necessary for the solvability of the $C_{i l}=0$. If $F$ has a modulus which divides $l-3$, the above determinant vanishes. Call $M_{2}, M_{3}, \cdots, M_{l-1}$ the minors of the elements $0, P_{32}, \cdots, P_{l_{12}}$. Then must $b_{l l}\left(P_{2 l} \dot{d}_{2}-P_{3 l} M_{3}+\cdots\right)=0$. The deterninant whose expansion is the second factor is seen as above to have the value 1.

It is unnecessary for our applications to inquire whether or not this restriction on $F$ is necessary for the validity of the theorem. 
3. If $F$ is a finite field the equation $a_{1} b_{11}^{2}+a_{2} b_{21}^{2}=1$ has solutions $b_{11}, b_{21}$ in $F$. Hence, applying the theorem of $\S 2$ for $n=2$, we see that any form $\sum_{i=1}^{n} a_{i} x_{i}^{2}$ in the $G F\left[p^{k}\right], p>2$, can be reduced by a succession of binary transformations to $\sum_{i=1}^{m-1} x_{i}^{2}+a x_{m}^{2}$. Since one-half of the marks, not zero, of the $G F\left[p^{k}\right], p>2$, are squares, while the ratio of any two not-squares is a square, we can make $a=1$ or a particular not-square $\nu$.

TheOREM.* In the GF $\left[p^{k}\right], p>2$, any m-ary quadratic form of nonvanishing determinant can be reduced to $\sum_{i=1}^{m} x_{i}^{2}$ or else to $\sum_{i=1}^{m-1} x_{i}^{2}+\nu x_{m}^{2}, \nu$ being a particular not-square.

4. Let $F$ be the field $R$ of all rational numbers. It $a_{1}=\alpha_{1} / d, a_{1} x_{1}^{2}=\alpha_{1} d y_{1}^{2}$, where $y_{1}=x_{1} / d$. Hence we may assume that each $a_{1}$ in (1) is an integer. If $a_{1}, \cdots, a_{4}$ are not all negative, the equation

$$
\sum_{i=1}^{4} a_{i} b_{i}^{2}=1
$$

can be satisfied by rational values of $b_{1}, \cdots, b_{4}$. Indeed, there exist $\uparrow$ integers $\beta_{1}, \cdots, \beta_{4}, \sigma$, not all zero, for which $\sum_{i=1}^{+} a_{i} \beta_{i}^{2}-\sigma^{2}=0$. But if $\sigma=0$ and say $\beta_{1} \neq 0, \beta_{2} \neq 0,(2)$ is satisfied by

$$
b_{1}=\frac{\beta_{1}}{2 \beta_{2}}\left(1-\frac{1}{a_{2}}\right), \quad b_{2}=\frac{1}{2}\left(1+\frac{1}{a_{2}}\right), \quad b_{3}=\frac{b_{1}}{\beta_{1}} \beta_{3}, \quad b_{4}=\frac{b_{1}}{\beta_{1}} \beta_{4} .
$$

It now follows that, if $a_{1}, \ldots, a_{1}$ are all negative, there exist rational solutions $b_{i}$ of $\sum a_{i} b_{i}^{2}=-1$. Hence, by the theorem of $\S 2, \sum_{i=1}^{4} a_{i} x_{i}^{2}$ can be transformed by a quaternary substitution with rational coefficients into

$$
\pm x_{1}^{2}+\sum_{i=2}^{4} a_{i}^{\prime} x_{i}^{2}
$$

the sign being + unless $a_{1}, \ldots, a_{4}$ are all negative. We thus obtain the

* The reduction of quadratic forms in the $G F\left[p^{k}\right]$ was effected by the writer in a memoir on the linear groups defined hy a quadratic invariant, A merican Journal of Matbematics, vol. 21 (1899), pp. 194, 222. (Cf. Linear Groups, pp. 158, 197.) For $p=2$, there exist $m$ ary quadratic forms of non-vanishing discriminant $D$ only for $m=2 n$, and then the two canonical types are

$$
F_{\lambda}=\lambda x_{1}^{2}+\lambda y_{1}^{2}+\sum_{i=1}^{n} x_{i} y_{i},
$$

where $\lambda=0$ or a particular one of the marks for which $\lambda x_{1}^{2}+\lambda y_{1}^{2}+x_{1} y_{1}$ is irreducible in the $G F\left[2^{k}\right]$. For $k=1$, JoRDAN states in his pemoir cited above that a quadratic form is reducible to $F_{0}$ or $F_{1}$ according as $\left(\frac{2}{D}\right)=+1$ or -1 . This is clearly an oversight since $D$ is the mark 1 and thus can be taken to be an arbitrary odd integer. The same oversight occurs in DE SÉGUIER's Groupes Abstraits, p. 51, footnote.

† A. MeYer, Vierteljahrsohrift der naturforschenden Gesellschaf t in Zurich, vol. 29 (1884), pp. 209-222. He shows that $a_{1} x_{1}^{2}+\cdots+a_{5} x_{6}^{2}=0$ has integral solutions $x_{i}$ not all zero if $a_{1}, \cdots, a_{5}$ are integers $\neq 0$, not all of like sign. 
Theorem. Within the field of all rational numbers any n-ary quadicatic form of non-vanishing determinant can be transformed into one of the forms

$$
-\sum_{i=1}^{\infty} x_{i}^{2}+\sum_{i=8+1}^{n-3} x_{i}^{2}+a x_{n-2}^{2}+b x_{n-1}^{2}+c x_{n}^{2},
$$

where $s=0$ unless $a, b, c$ are all negative.

The difficult problem of ultimate canonical forms is not undertaken here. I pass to hermitian forms and effect a complete reduction to canonical forms.

\section{On the reduction of hermitian forms.}

5. Let $F^{\prime}$ be any field* for which there is an equation

$$
x^{2}+u x+v=0
$$

$(\operatorname{roots} \omega, \bar{\omega}, \bar{\omega} \neq \omega) \dagger$,

belonging to and irreducible in $F$. Denote the field $F(\omega)$ by $Q$. Any element of $Q$ may be given the form $e=a+b \omega, a$ and $b$ in $F$. Set $\bar{e}=a+b \omega$. Then

$$
H_{a} \equiv \sum_{i, j}^{1, \ldots n} \alpha_{i j} \xi_{i} \xi_{j} \quad\left(\text { each } \alpha_{i j} \text { in } Q, \bar{\alpha}_{i j}=\alpha_{i i}\right)
$$

will be called a hermitian.form in the field $Q$, and $\left|\alpha_{i j}\right|$ will be called the determinant of $H_{a}$. Under a linear transformation

$$
\xi_{i}=\sum_{k=1}^{n} \beta_{i k} \eta_{k i}, \quad \xi_{i}=\sum_{k=1}^{n} \bar{\beta}_{i k} \bar{\eta}_{k k} \quad(i=1, \cdots, n),
$$

in which the $\beta_{i k}$ are elements of $Q$ and $B \equiv\left|\beta_{i k}\right| \neq 0, H_{u}$ becomes a hermitian form $H_{\gamma}$ whose determinant. $\left|\gamma_{i j}\right|$ equals $B \bar{B}\left|\alpha_{i j}\right| \cdot+$

6. Th Fonem. By a transformation (3) in $Q$ of determinant unity, any hermitian form $H_{a}$ in $Q$ of non-vanishing determinant $\Delta$ can be reduced to

$$
\sum_{i=1}^{n} \gamma_{i} \eta_{i} \bar{\eta}_{i}
$$

$\left(\right.$ each $\gamma_{i}$ in $\left.F\right)$.

We first reduce $H_{\alpha}$ to a form $H_{a^{\prime}}$ having $\alpha_{11}^{\prime} \neq 0$. If $\alpha_{11}=0$ and $\alpha_{j j} \neq 0$, we apply the transformation $\xi_{1}=\eta_{j}, \xi_{j}=-\eta_{1}$. If every $\alpha_{i i}=0$, we may take $\alpha_{12} \neq 0$; under the transformation $\xi_{1}=\eta_{1}, \xi_{2}=\eta_{2}+\mu \eta_{1}, H_{a}$ becomes $H_{a^{\prime}}$ with

* In particular, $F$ shall not be the field of all complex numbers $x+y i$, nor a field $F_{m, \text {, }}$ defined as the aggregate of the Galois fields of orders $p^{m}, p^{2 m}, p^{4 m}, \mu^{8 m}, \ldots$. If in a field $F$ of modulus 2 every equation $x^{2}+u x-v(u \neq 0)$ is reducible, $F$ contains $F_{1,2}$.

$\dagger$ In the field of all rational functions of a variable $z$ with integral coefficients taken modulo 2, the equation $x^{2}=z$ is irreducible but has equal roots. We exclude such a case $\bar{\omega}=\omega$, since the problem is then that of bilinear forms in $F$ subject to cogredient transformations.

$\ddagger$ The simplest proof follows by use of generators of the types

$$
\xi_{1}=\eta_{1}+i_{1}, \xi_{i}=\eta_{i} \quad \text { and } \quad \xi_{1}=B \eta_{1}, \xi_{i}=\eta_{i}
$$


$\alpha_{11}^{\prime}=\alpha_{12} \bar{\mu}+\bar{\alpha}_{12} \mu$. Take $\mu=(a+b \omega.) / \bar{x}_{12}$; then $\alpha_{11}^{\prime} \equiv 2 a-b u$ can be made different from zero, since we have excluded the case $u=0$ when $F$ has modulus 2 by assuming that $\bar{\omega} \neq \omega$.

In $H_{a^{\prime}}$ with $\alpha_{11}^{\prime} \neq 0$, set $\xi_{1}=\eta_{1}-\bar{\alpha}_{12}^{\prime} \alpha_{11}^{\prime-1} \eta_{2}, \xi_{2}=\eta_{2}$; there results $H_{a^{\prime \prime}}$ with $\alpha_{11}^{\prime \prime}=\alpha_{11}^{\prime}, \alpha_{12}^{\prime \prime}=\alpha_{21}^{\prime \prime}=0$. Similarly, we make every $\alpha_{1 i}$ and $\alpha_{i 1}$ zero $(i>1)$ and reach $H_{\alpha^{\prime \prime}}=\alpha_{11}^{\prime} \eta_{1} \bar{\eta}_{1}+f^{\prime}$, where $f$ is a hermitian form on $\eta_{i}, \bar{\eta}_{i}(i=2, \cdots, n)$ of determinant $\neq 0$.

7. Let $F^{\prime}$ be the $G F\left[p^{\prime \prime \prime}\right]$, so that $Q$ is the $G F\left[p^{2 m}\right]$. For any mark $g$ of $F$ the equation $c \bar{c}=g$, viz., $c^{c^{\prime m+1}}=g$, is solvable in $Q$. Hence there is an unique canonical form $\Sigma \xi_{i} \bar{\xi}_{i}$.

8. Let $F$ be the field of all real numbers and set $\omega=V-1$. Then $\gamma_{i}= \pm c_{i}^{2}$, so that (4) can be reduced to one of the forms

$$
h_{r} \equiv \sum_{i=1}^{r} \xi_{i} \bar{\xi}_{i}-\sum_{i=r+1}^{n} \xi_{i} \bar{\xi}_{i} \quad(r=0,1, \cdots, n) .
$$

Now $r$ is an invariant, i. e., $h_{r}$ can not be reduced to $h_{\rho}(\rho \neq r)$ by a transformation (3). Indeed, for $\xi_{i}=x_{i}+y_{i} \sqrt{-1}$, (3) becomes a special $2 n$-ary real linear transformation, and (5) becomes

$$
\sum_{i=1}^{r}\left(x_{i}^{2}+y_{i}^{2}\right)-\sum_{i=r+1}^{n}\left(x_{i}^{2}+y_{i}^{2}\right),
$$

so that $2 r$ is an invariant by $\S 1$. There are $n+1$ canonical forms (5).

9. Consider for a general field $F^{\prime}$ the possible normalizations of a binary hermitian form $h=a(x \bar{x}+r y \bar{y}), a$ and $r$ in $F$, and each $\neq 0$. Set

$$
x=\lambda X+\mu Y, \quad y=\rho X+\sigma Y, \quad D \equiv \lambda \sigma^{+}-\mu \rho \neq 0 .
$$

Then $h$ becomes

$$
h^{\prime}=a(\lambda \bar{\lambda}+r \rho \rho) X \bar{X}+a(\mu \bar{\mu}+r \sigma \bar{\sigma}) Y \bar{Y}+A X \bar{Y}+\bar{A} \bar{X} Y,
$$

where $A=a(\lambda \bar{\mu}+r \rho \bar{\sigma})$. We desire that $A=0$. For $\lambda \neq 0$, we take $\mu=-r \bar{\rho} \sigma / \bar{\lambda}$. Then $D=(\lambda \bar{\lambda}+r \rho \bar{\rho}) \sigma / \bar{\lambda}$, and

$$
h^{\prime}=a(\lambda \bar{\lambda}+r \cdot \rho \bar{\rho})(X \bar{X}+r \cdot \bar{\tau} Y \bar{Y}), \quad \tau \equiv \underset{\lambda}{\sigma} .
$$

The same form with $\tau=\mu / r \rho$ results if $\lambda=0$, whence $\sigma=0$. Hence $r$ can be changed only by a factor $\tau \bar{\tau}$. By a preliminary unary transformation on $y$, we can restrict $r$ to the series of multipliers $1, m_{1}, m_{2}, \ldots$ in a rectangular array of the elements of $F$ with the various distinct elements $1, \kappa_{1} \bar{\kappa}_{1}, \kappa_{2} \bar{\kappa}_{2}, \ldots$ in the first row, where the $\kappa$ 's are arbitrary in $Q$. To retain this norinalization, we set $\tau=1$. 
TheORem. Let $1, m_{1}, m_{2}, \ldots$ be the multipliers in a rectangular array of elements of $F$, the elements of the first row being the distinct elements $\kappa \bar{\kappa}, \kappa$ ranging over $Q$. Any binary hermitian form can be reduced linearly to to $a(x \bar{x}+r y \bar{y})$, where $a$ and $r$ belong to the set $1, m_{1}, m_{2}, \ldots$ Two such reduced forms can be transformed into each other if and only if they have the same $r$, and the ratio of their $a^{\prime} s$ is a mark, not zero, expressible in the form $\lambda \lambda+r \rho \bar{\rho}$.

10. Let $F$ be the field $R$ of all rational numbers. Then $Q=R(\epsilon)$, where $\epsilon^{2}=\nu, \nu$ being a fixed integer $\neq 1$ having no square factor. Thus $\bar{\epsilon}=-\epsilon$. By $\S 9$, we can transform $a x \bar{x}+b y \bar{y}$ into $L x \bar{x}+M y \bar{y}$, where $L=a \lambda \bar{\lambda}+b \rho \bar{\rho}$, $\lambda$ and $\rho$ being any elements of $Q$ for which $L \neq 0$. If $a=\alpha / d, \alpha$ and $d$ being integers, then $a x \bar{x}=\alpha d z \bar{z}, z=x / d$. Hence we may assume that $a$ and $b$ are integers. Set $\lambda=\alpha+\beta \epsilon, \rho=\gamma+\delta \epsilon, \alpha, \beta, \gamma, \delta$ in $R$. Then

$$
L \equiv a \lambda \bar{\lambda}+b \rho \bar{\rho}=a\left(\alpha^{2}-\nu \beta^{2}\right)+b\left(\gamma^{2}-\nu \delta^{2}\right) .
$$

As* in $\$ 4$, we can choose $\alpha, \beta, \gamma, \delta$ in $R$ to make $L=+1$ if $\nu, a, b$ are not all negative, and $L=-1$ in the contrary case. Hence if $\nu$ is positive, any $n$-ary hermitian form is reducible to

$$
\sum_{i=1}^{n-1} \xi_{i} \xi_{i}+r \xi_{n} \bar{\xi}_{n}
$$

while, if $\nu$ is negative, it can be reduced to one of the types

$$
f_{.} \equiv-\sum_{i=1}^{8} \xi_{i} \xi_{i}+\sum_{i=s+1}^{n-1} \xi_{i} \bar{\xi}_{i}+r \xi_{n} \bar{\xi}_{n} \quad(s=0,1, \cdots, n-1)
$$

If $r>0$ and $s>0$, we can transform $-\xi_{s} \xi_{s}+r \xi_{n} \xi_{n}$ into $+\xi_{8} \xi_{8}+r^{\prime} \xi_{n} \xi_{n}$, and hence transform $f_{s}$ into $f_{s-1}$. The reduced forms for $\nu$ negative are thus

$$
f_{0} \text { with } r>0 ; f_{0}(s=0,1, \ldots, n-1) \text { with } r<0 \text {, }
$$

falling into $n+1$ types, each characterized by the number of its negative terms. Hence by $\S 8$, a form of one type cannot be reduced to one of a different type by a transformation (3). But by $\S 5$, under a transformation (3) of determinant $B$, the determinant $(-1)^{s} r$ of $f_{s}$ is multiplied by $B \bar{B}$. Hence $f_{s}(r)$ is reducible to $f_{v}(\rho)$ if and only if $\rho / r$ is expressible in the form $B \bar{B}$.

THEOREM. Any n-ary hermitian form in $R(\sqrt{\nu})$ with non-vanishing determinant can be reduced by a linear transformation in $R(\sqrt{\nu})$ to one and but one of the canonical forms :

*.The case $\sigma=0$ may now be treated more naturally. There are then solutions $\lambda_{1} \neq 0, \rho_{1} \neq 0$ in $R(\varepsilon)$ of $a \lambda_{1} \bar{\lambda}_{1}+b \rho_{1} \bar{\mu}_{1}=0$. Let $\tau=: \rho \lambda_{1} / \rho_{1}$. Then $L=1$ becomes $\lambda \bar{\lambda}-\tau \tau=a^{-1}$, and is satisfied by $\lambda=\frac{1}{2}\left(a^{-1}+1\right), \tau=\frac{1}{2}\left(a^{-1}-1\right)$. 
(I) for $\nu>0, \sum_{i=1}^{n-1} \xi_{i} \bar{\xi}_{i}+r \xi_{n} \bar{\xi}_{n}$, where $r$ ranges over the multipliers (integers) $1, m_{1}, m_{2}, \ldots$, in a rectangular array of all rational numbers with those representable by $x^{2}-\nu y^{2}$ in the first row;

(II) for $\nu<0, \sum_{i=1}^{n-1} \xi_{i} \xi_{i}+\rho \xi_{n} \xi_{n},-\sum_{i=1}^{*} \xi_{i} \xi_{i}+\sum_{i=x+1}^{n-1} \xi_{i} \xi_{i}-\rho \xi_{n} \xi_{n}$, where $s=0,1, \ldots, n-1$, and $\rho$ ranges over the multipliers (positive integers) in a rectangular array of all positive rational numbers with those representable by $x^{2}-\nu y^{2}$ in the first row.

In the examples,* $(k)$ denotes all primes of the form $k ; q_{1}, q_{2}, q_{3}, \ldots$, denote distinct primes; $p_{1}, p_{2}, p_{3}, \ldots$, denote distinct primes.

For $\nu=2, r=1, q_{1}, q_{1} q_{2}, q_{1} q_{2} q_{3}, \cdots, q_{i}$ ranging over $(8 m+3),(8 m+5)$.

For $\nu=3, r= \pm 1, \pm q_{1}, \pm q_{1} q_{2}, \cdots, q_{i}$ ranging over $(12 m+5),(12 m+7)$.

For $\nu=5, r=1, q_{1}, q_{1} q_{2}, \cdots, q_{i}$ ranging over $(20 m+3),(20 m+7)$, $(20 m+13),(20 m+17)$.

For $\nu<0, \rho=1, q_{1}, q_{1} q_{2}, q_{1} q_{2} q_{3}, \cdots$, where for $\nu=-1, q_{i}=(4 m+3)$; for $\nu=-2, q_{i}=(8 m+5),(8 m+7)$; for $\nu=-3, q_{i}=(3 m+2)$; for $\nu=-5$, the only limitations on the primes $q_{i}$ are that no one is $5,20 m+1$, or $20 m+9$, while at most one is chosen from the set $2,(20 m+3),(20 m+7)$; for $\nu=-6$, no $q_{i}$ is $24 m+1$ or $24 m+7$, while at most one is chosen from the set $2,3,(24 m+5),(24 m+11)$; for $\nu=-7, q_{i}=(28 m+t), t=3,5$, $13,17,19,27$.

\section{The bilinear forms invariant under a given substitution $S$.}

11. Let $F$ be an arbitrarily given field. We seek all bilinear functions $\Phi=\sum_{i j} \gamma_{i j} \xi_{i} \eta_{j}$ with discriminant $D \equiv\left|\gamma_{i j}\right| \neq 0$ and coefficients in $F$, such that $\Phi$ is invariant under a given substitution, cogredient in the two sets of variables,

$$
S: \quad \xi_{i}^{\prime}=\sum_{j=1}^{n} \alpha_{i j} \xi_{j}, \quad \eta_{i}^{\prime}=\sum_{j=1}^{n} \alpha_{i j} \eta_{j} \quad(i \cdots 1, \cdots, n),
$$

with coefficients also in $F$. In the canonical form of $S$ (with the initial variables $\xi_{i}$ ), the new variables fall into as many classes as there are distinct roots of the characteristic equation $\Delta(\rho)=0$ of $S$. Each class is composed of one or more series, the variables of any series being transformed by $S$ into linear functions of themselves, as follows :

$$
\left|x_{0}, x_{1}, \cdots, x_{t} \quad \rho x_{0}, \rho\left(x_{1}+x_{0}\right), \cdots, \rho\left(x_{t}+x_{t-1}\right)\right| .
$$

* The integers representable by $x^{2}-2 y^{2}$ are, aside from square factors, $\pm 1, \pm 2,(8 n+1)$, $(8 n+7)$, and their products two, three, four. $\cdots$, at a time. For $x^{2}-3 y^{2}$, they are $-2,-3$, $(12 m+1),-(12 m+11)$. For $x^{2}+5 y^{2}$, they are $5,(20 m+1),(20 m+9), 2 p_{1}, p_{1} p_{2}$, and the products of these expressions two, three, $\cdots$, at a time, where $p_{i}=(20 m+3),(20 m+7)$. For $x^{2}+6 y^{2}$, they are $(24 m+1),(24 m+7), p_{1} p_{2}$, and their products, where $p_{i}=2,3,24 m+5$ or $24 m+11$. 
Similarly, $S$ with the initial variables $\eta_{i}$ affects the general series of canonical variables as follows :

$$
\left|y_{0}, y_{1}, \cdots, y_{\tau} \quad \rho_{1} y_{0}, \rho_{1}\left(y_{1}+y_{0}\right), \cdots, \rho_{1}\left(y_{\tau}+y_{\tau-1}\right)\right| \text {. }
$$

In the new variables, $\Phi$ becomes a sum of functions each separately invariant, the general one, $f$, being bilinear in $x_{i}, y_{j}(i=0, \ldots, t ; j=0, \ldots, \tau)$. Let $a x_{i} y_{j}$ be any term of the latter, $i+j$ its rank, $i$ being the rank of $x_{i}$ and $j$ the rank of $y_{j}$. Since the increment obtained from any term is of lower rank, the set of terms of maximum rank in the transform of $f$ by $S$ is derived from the set of terms of maximum rank in $f$ by multiplication by $\rho \rho_{1}$. Unless $f$ is identically zero, $\rho \rho_{1}=1$. But if none of the variables $x$ appeared in the new form of $\Phi$, its discriminant would vanish. Hence there is at least one series of variables $y$ which $S_{\eta}$ multiplies by $\rho^{-1}$. With the class $C_{x}$ of all the variables which $S_{\xi}$ multiplies by $\rho$ is associated a class $C_{y}$ of the variables which $S_{\eta}$ multiplies by $\rho^{-1}$. Then $\Phi=\left[C_{x} C_{y}\right]+\Psi$, where $\left[C_{x} C_{y}\right]$ is bilinear in the variables of $C_{x}, C_{y}$, while $\Psi$ does not contain those variables. The discriminant of $\left[C_{. x} C_{y}\right]$ is a factor of $D$ and hence is not zero; this requires that the classes $C_{. r}$ and $C_{n}$ shall contain the same number of variables. Thus $\rho^{-1}$ must be a root of $\Delta(\rho)=0$ of the same multiplicity as the root $\rho$. A first necessary condition for an invariant $\Phi$ under $S$ is :

(11) The characteristic equation of $S$ must be a reciprocal equation.

A second condition for the existence of $\left[C_{. r} C_{y}\right]$ is (Jokdan, $\S 13$ ):

(12) Classes $C_{x}, C^{y}$ must be of like type as to number and length of series.

12. $\mathrm{W}^{\top}$ hen these conditions on $S^{\mathrm{r}}$ are satisfied, invariants $\left[C_{*} C_{y}\right]$ of nonvanishing discriminant exist (JoRDAN, § 12, case I alone occurs for bilinear functions), the general one being $\sum_{a, \beta, r} a_{r}^{a \beta} f_{a \beta r}$, where $f$ is a definite bilinear function and the $a$ 's are any polynomials in $\rho, \rho_{1}, \cdots, \rho_{\nu-1}$, for which the discriminant of $\left[C_{x} C_{y}\right]$ is not zero and the following " reality" conditions hold : Since $\Phi$ is to be equal to a function of the initial variables with coefficients in $F$, we must have $\Phi=\Sigma+\Phi_{1}$, where

$$
\Sigma=\left[C_{x} C_{y}\right]+\left[C_{x}^{\prime} C_{y}^{\prime \prime}\right]+\cdots+\left[C^{\nu-1} C_{y}^{\nu-1}\right],
$$

$\left[C_{. r}^{\prime} C_{y}^{\prime}\right], \cdots$, being derived from $\left[C_{x} C_{y}\right]$ by interchanging $\rho$ and $\rho_{1}, \cdots$, $\rho$ and $\rho_{v^{\prime}-1}$, respectively, $\rho_{1}, \cdots, \rho_{\nu-1}$, being the remaining roots of the same irreducible factor of $\Delta(\rho)$. Thus $\alpha_{r}^{\alpha \beta}$ must equal a polynomial in $\rho$. Let these conditions be satisfied. Then (JoRdAN, $\left.{ }^{*} \$ 14-16\right)$ by a linear transformation leaving the canonical form of $S$ unaltered (not necessarily the same transformation. on the $y$ 's as on the $x$ 's), we can reduce $\Phi$ to a unique canonical form.

* An obvious correction (not altering the argument) is to be made on p. 237, 1. 11. The expression in brackets should be

$$
y_{0}^{\prime}\left(x_{m}^{\prime}+C_{m-1}^{1} x_{m-1}^{\prime}+\cdots+x_{1}^{\prime}\right)+y_{0}^{\prime \prime}\left(x_{m}^{\prime \prime}+C_{m-1}^{1} x_{m-1}^{\prime \prime}+\cdots+x_{1}^{\prime \prime}\right)+\cdots
$$


13. Conditions (11) and (12) depend upon quantities irrational in general with respect to the initial field $F$. It seems desirable to proceed further and exhibit purely rational conditions for the solvability of the problem. We obtain * the

THeOREM. The necessary and sufficient conditions that a given substitution $\left(\alpha_{i j}\right)$ with coefficients in $F$ shall leave invariant one or more bilinear forms with coefficients in $F$ are: (i) the characteristic determincant $\Delta(\rho)$ or $S$ hus a decomposition into factors irreducible in $F$ of the type

$$
\Delta(\rho)=S_{l i}^{\prime \prime} S_{1}^{\prime b} \ldots R_{i}^{\prime} R_{i}^{* *} \ldots R_{*}^{\prime \prime} R_{*}^{\prime * \prime \prime} \ldots,
$$

where $S_{k}, S_{1}^{\prime}, \ldots$ are self-reciprocal, viz., $S_{k}(\rho) \equiv \rho^{k} S_{k}\left(\rho^{-1}\right)$, while $R_{\text {, and }}$ $I_{*}^{*}$, are reciprocal; (ii) the invariant-factors of the matrix $\Delta(\rho)$ are of the form

$$
S_{l}^{a_{1}} S_{l}^{\prime b_{1}} \cdots R_{t}^{e_{1}} R_{t}^{* *_{1}} \cdots R_{s}^{\prime f_{1}} R_{*}^{\prime * f_{1}}, S_{k}^{a_{2}} S_{l}^{b_{2}} \ldots R_{t}^{*_{s}} R_{t}^{* *_{2}} \cdots R_{s}^{\prime f_{2}} R_{s}^{\prime * f_{2}}, \cdots,
$$

a pair of reciprocal fuctors occurring always to the same power.

When these conditions are satisfied there exist in $F$ bilinear forms $\sum b_{i j} \xi_{i} \eta_{j}$ invariant under $S_{\xi}, S_{\eta}$. All such invariunt forms are reducible to a single one by a transformation on the $\xi$ 's commutation with $S_{\xi}$, and a (possibly different) transformation on the $\eta$ 's commutative with $S_{\eta}$.

IV. The quadrutic forms incariunt under a. given substitution $S$.

14. Jordan's treatment of the case of a field of order $p$, a prime, can be extended immediately to any finite field, and with certain essential modifications to any infinite field. Let $F$ be any field.

Let $\rho$ be a root of $f(x)=0$, where $f(x)$ is a factor of degree $k$ of $\Delta(x)$ and is irreducible in $F$. If $\rho^{-1}$ is not a root of $f(x)=0$, the question is essentially the same as that for bilinear forms, discussed above. Let next $\rho^{-1}$ be a root of $f(x)=0, \rho^{-1} \neq \rho$. Then $f(x)=0$ and $x^{k} f\left(x^{-1}\right)=0$ are equations belonging to and irreducible in $F$ with a root $\rho$ in common; hence all their roots are common and $f(x)=0$ is a reciprocal equation. Since no root equals its reciprocal, $k=$ even $=2 \nu$, and the roots may be designated $\rho \equiv \rho_{0}, \rho_{1}, \ldots, \rho_{2, v-1}$ with $\rho_{v}=\rho_{0}^{-1}, \rho_{v^{\prime}+1}=\rho_{1}^{-1}, \ldots, \rho_{2 \nu-1}=\rho_{r^{\prime}-1}^{-1}$. In the canonical form of $S$ all the variables corresponding to a ront $\rho_{i}$ are said to form the class $C_{i}$. Thus an invariant quadratic form $\Phi$ must equal $\left[C_{0} C_{\nu}\right]+\Psi$, where $\left[C_{0} C_{\nu}\right]$ is bilinear in the variables of classes $C_{0}$ and $C_{\nu}$, while $\Psi$ does not contain them: Now $\left[C_{0}^{\gamma} C_{v}\right]$ which is itself invariant must, have the form

$$
\left[C_{0} C_{\nu}\right]=\sum_{a, \beta, r} a_{r}^{\alpha \beta} F_{a \beta},
$$

where $a_{r}^{\alpha \beta}$ are any constants satisfying the conditions later specified, while $F_{a \beta r}$

* Cf. Transactions, vol. 3 (1902), pp. 290-292. 
are perfectly definite bilinear forms derived from those given by JORDAN, pp. 240,241 , by making the following changes. For $b_{i}, b_{i}^{p^{\nu}}, c_{i}, c_{i}^{p^{\nu}}, \ldots$ write $b_{i}(\rho), b_{i}\left(\rho^{-1}\right), c_{i}(\rho), c_{i}\left(\rho^{-1}\right), \cdots$, respectively; for $e$ of $\S 21$ write $\rho-\rho^{-1}$. In $\S 20$, take $b_{0}=\frac{1}{2}$ if $F$ does not have modulus 2 , while for modulus 2 take

$$
b_{0}=-\frac{1}{\alpha_{\nu}}\left(\rho^{\nu}+\alpha_{1} \rho^{\nu-1}+\alpha_{2} \rho^{\nu-2}+\cdots+\alpha_{\nu-1} \rho\right),
$$

where the $\alpha$ 's refer to the irreducible reciprocal equation with root $\rho$ :

$$
E \equiv y^{\nu}+y^{-\nu}+\alpha_{1}\left(y^{\nu-1}+y^{-\nu+1}\right)+\cdots+\alpha_{\nu-1}\left(y+y^{-1}\right)+\alpha_{\nu}=0 .
$$

If $a_{v}$ vanished, there would be a factor $y-y^{-1}$ modulo 2 . Since the variables entering $F_{a \beta r}$ are linear functions of the initial variables $\xi_{i}$ whose coefficients are polynomials in $\rho$ with coefficients in $F, F_{a \beta r}$ can be expressed as a function of $\rho$ and the $\xi$ 's with coefficients in $F$. In particular $F_{a \beta r}$ is unaltered by any permutation of the roots $\rho_{1}, \rho_{1}^{-1}, \ldots, \rho_{\nu-1}, \rho_{\nu-1}^{-1}$. The same must be true of $\left[C_{0} C_{\nu}\right]$, which is composed of all the terms of $\Phi$ involving the variables of classes $C_{0}$ and $C_{\nu}$ (viz., those corresponding to the roots $\rho$ and $\rho^{-1}$ ), since $\Phi$ is to be equal to a function of the $\xi$ 's with coefficients in $F$. Hence the $a$ 's in (13) must be symmetric functions of those $2 \nu-2$ roots; but the latter are the roots of $E /\left(y+y^{-1}-\rho-\rho^{-1}\right)=0, E$ being given by (14). Hence the coefficients in (13) are rational functions of $\rho$ with coefficients in $F$. By its construction $F_{a \beta r}$ becomes $F_{B a r}$ when $\rho$ is replaced by $\rho^{-1}$. Hence

$$
a_{r}^{\alpha \beta}(\rho)=a_{r}^{\beta a}\left(\rho^{-1}\right),
$$

whence $a_{r}^{\alpha a}$ is a polynomial in $\rho+\rho^{-1}$.

Finally, since $\left[C_{0} C_{v}\right]+\Psi$ is to be equal to a function $\Phi$ of the $\xi$ 's with coefficients in $F, \Psi$ must contain the bilinear forms $\left[C_{1} C_{\nu+1}\right], \cdots,\left[C_{\nu-1} C_{2 v-1}\right]$ derived from $\left[C_{0} C_{\nu}\right]$ by replacing $\rho$ by $\rho_{1}, \cdots, \rho_{\nu-1}$, respectively. Hence must $\Phi=Q+\Phi_{1}$, where $\Phi_{1}$ involves no variable in the classes $C_{0}, \ldots, C_{2 v-1}$, while

$$
Q \equiv \sum_{i=0}^{\nu-1}\left[C_{i} C_{\nu+i}\right]=\sum_{a, \beta, r, i} a_{r}^{\alpha \beta}\left(\rho_{i}\right) F_{a \beta r}^{(i)},
$$

$F_{a \beta r}^{(i)}$ being the same function of $\rho_{i}$ and the variables of $C_{i}, C_{v+i}$ that $F_{\alpha \beta r}^{0} \equiv F_{a \beta r}$ is of $\rho_{0}$ and the variables of $C_{0}, C_{\nu}$. In (16), the $u$ 's are arbitrary rational functions of $\rho_{i}$ satisfying (15) and making the descriminant of $\left[C_{0} C_{v}\right]$ not zero (requiring that certain determinants of the $a$ 's be $\neq 0$ ). The same argument is to be repeated for $\Phi_{1}$ with reference to each new irreducible factor of $\Delta(\rho)$.

15. The next problem is the reduction of $\left[C_{0} C_{\nu}\right]$ to one or more normal forms by means of a transformatian of variables leaving $S$ unaltered. JoRDAN shows (\$§ 23-28) that a unique normal form results when $F$ is a field of order 
$p$. The same is true for any finite field, but not for an arbitrary infinite field. For an arbitrary field $F$ we can proceed with JoRDAN's normalization by observing the following modifications.* The constants $a, a^{\mu^{\nu}}, \lambda, \lambda^{p^{\nu}}, \cdots$ are to be interpreted as $a(\rho), a\left(\rho^{-1}\right), \lambda(\rho), \lambda\left(\rho^{-1}\right), \cdots$, respectively.

To prove that the argument at the bottom of p. 243 remains valid, we have to show that, for $a_{m}^{21} \neq 0$,

$$
\lambda(\rho) a_{m}^{21}(\rho)+\lambda\left(\rho^{-1}\right) a_{m}^{12}(\rho)+\lambda(\rho) \lambda\left(\rho^{-1}\right) a_{m}^{22}(\rho)
$$

is not identically zero for every rational function $\lambda$. But if the sum vanished for $\lambda(\rho)=1,-1$, and $\rho$, then would

$$
\left|\begin{array}{rrr}
1 & 1 & 1 \\
-1 & -1 & 1 \\
\rho & \rho^{-1} & 1
\end{array}\right| \equiv-2\left(\rho^{-1}-\rho\right)=0,
$$

which is impossible if $F$ does not have modulus 2 . For modulus 2, we employ $\lambda(\rho)=1, \rho$, and $\rho+1$, obtaining as the determinant $\left(\rho^{-1}+\rho\right)\left(\rho^{-1}-\rho\right)$.

The argument to make $a_{m}^{11}=1$ (top of p. 244) must now be abandoned. For the present we allow $a_{m}^{11}$ to remain arbitrary, but $\neq 0$. To make $a_{m}^{12}=a_{m}^{21}=0$, apply the transformation which replaces $x_{i}^{\prime}$ and $y_{i}^{\prime}$ by $x_{i}^{\prime}-a(\rho) x_{i}^{\prime \prime}$ and $y_{i}^{\prime}-a\left(\rho^{-1}\right) y_{i}^{\prime \prime}$, respectively, for $i=m, \cdots, 0$, taking $a(\rho)=u_{m}^{21}(\rho) / a_{m}^{11}(\rho)$. By analogous transformations we can make every $a_{m}^{\alpha \beta}=0(\alpha \neq \beta)$ and reach

$$
\phi_{a}=A \sum_{a=1}^{l}\left\{a_{m}^{\alpha a}\left[x_{m}^{a} y_{m}^{a}+(-1)^{m} x_{m}^{a} y_{0}^{a}\right]\right\} \quad\left(\text { each } a_{m}^{a a} \neq 0, A \neq 0\right) .
$$

Consequently we insert the factprs $\alpha_{m}^{\alpha a}$ in the formulæ of $\$ \S 26-28$. For $\lambda$ on p. 248 we now take

$$
\lambda(\rho)=-c\left(\rho^{-1}\right) \div\left\{A\left(\rho^{-1}\right) u_{m}^{a a}(\rho)\right\} .
$$

For case $1^{\circ}$, p. 250 , the condition to be satisfied is now

$$
(-1)^{m \prime^{\prime}-r^{\prime}}\left[\lambda\left(\rho^{-1}\right)+\lambda(\rho)\right] a_{m}^{a a}+\omega_{r}^{a a}=0,
$$

where each $a$ is a rational function of $\rho+\rho^{-1}$. To this end we apply the

Lемм. If $\rho$ is a root of an irreducible reciprocal equation (14), we can determine a polynomial $\lambda(\rho)$ such that $\lambda\left(\rho^{-1}\right)+\lambda(\rho)=f\left(\rho+\rho^{-1}\right), f^{\prime}$ being any given polynomial, where the coefficients of $\lambda$ and $f$ belong to the arbitrary field $F$.

In view of (14), we may set

$$
f=g_{1}\left(\rho^{\nu-1}+\rho^{-\nu+1}\right)+\cdots+g_{\nu-1}\left(\rho+\rho^{-1}\right)+g_{\nu} \quad\left(g^{\prime} \text { in } F\right) .
$$

* We do not consider the numerical results of $\$ \S 24,25$, peculiar to finite tields. On p. 245 , line 6, the term $c_{1}^{p_{1}^{\nu}} d_{1}$ should be deleted. The group considered in ?.24 occurs in the literature, Mathematische Annalen, vol. 52 (1899) p. 561, and vol. 55 (1902), p. 521, as the hyperorthogonal group in the $G F\left[p^{2 \nu}\right]$. 
We proced to exhibit a solution $\lambda$ of the form

$$
\lambda(\rho)=c_{0} \rho^{\nu}+c_{1} \rho^{\nu-1}+\cdots+c_{\nu}
$$

If $F$ does not have modulus 2 we may take

$$
c_{0}=0, c_{1}=g_{1}, \cdots, c_{\nu-1}=g_{v-1}, c_{\nu}=\frac{1}{2} g_{v} .
$$

If $F^{\prime}$ has modulus 2, we apply (14) to eliminate $\rho^{\nu}+\rho^{-v}$ and get

$$
\lambda\left(\rho^{-1}\right)+\lambda(\rho)=\sum_{i=1}^{\nu-1}\left(c_{i}-c_{0} \alpha_{i}\right)\left(\rho^{\nu-i}+\rho^{-\nu+i}\right)-c_{0} \alpha_{\nu} .
$$

Hence this equals $f$ if we set

$$
c_{0}=\frac{g_{v}}{\alpha_{\nu}}, \quad c_{i}=g_{i}+\frac{\alpha_{i} g_{v}}{\alpha_{v}} \quad(i=1, \cdots, v-1) .
$$

For case $2^{\circ}$, page 250 , the condition to be satisfied is now *

$$
(-1)^{m^{\prime}-r^{\prime}-1} e\left[\lambda\left(\rho^{-1}\right)-\lambda(\rho)\right] a_{m}^{a a}+a_{r}^{a a}=0, e \equiv \rho-\rho^{-1} .
$$

Now $(-1)^{m^{\prime}-r^{\prime}} a_{r}^{a a} / a_{m}^{a a}$ equals a polynomial $f\left(\rho+\rho^{-1}\right)$ by (15). Set $\mu(\rho)=\left(\rho^{-1}-\rho\right) \lambda(\rho)$. The resulting condition $\mu\left(\rho^{-1}\right)+\mu(\rho)=f\left(\rho+\rho^{-1}\right)$ may be satisfied by the Lemma.

Cases $3_{1}^{\circ}, 3_{2}^{\circ}$ are analogous to $2^{\circ}, 1^{\circ}$, respectively.

Hence $\left[C_{0} C_{\nu}\right]$ can be reduced to the semi-normal form

$$
\sum_{a=1}^{l} a_{m}^{a_{2}} F_{\alpha a m}+\sum_{\beta=l+1}^{l+l^{\prime}} a_{m^{\prime}}^{\beta \beta} F_{\beta \beta m^{\prime}}+\sum_{\gamma=l+l^{\prime}+1}^{l+l^{\prime}+l^{\prime \prime}} a_{m^{\prime \prime}}^{\gamma \gamma} F_{\gamma \gamma m^{\prime \prime}}+\cdots,
$$

where each $a=a(\rho)=a\left(\rho^{-1}\right) \neq 0$, while $F_{\text {aam }}$ is a bilinear function with fixed coefficients of $x_{i}^{a}$ and $y_{i}^{a}(i=0,1, \ldots, m ; \alpha=1, \cdots, l), F_{\beta \beta m^{\prime}} a$ bilinear function of $x_{i}^{\beta}$ and $y_{i}^{\beta}\left(i=0,1, \ldots, m^{\prime} ; \beta=l+1, \ldots, l+l^{\prime}\right)$, etc. Also $y(\rho)=x\left(\rho^{-1}\right), m>m^{\prime}>m^{\prime \prime} \cdots$.

16. First let $F$ be a finite field, the $G F\left[p^{k}\right]$. Then $F(\rho)$ is the $G F\left[p^{2 \nu k}\right]$ and $\rho^{p^{\nu k}}=\rho^{-1}$ The $a$ 's belong to the $G F\left[p^{\nu k}\right]$. Apply the transformation (commutative with $S$ ) which multiplies each $x_{i}^{a}$ by $\lambda$ and each $y_{i}^{a}$ by $\lambda^{p^{\nu k}}$. The new coefficients of $F_{a a m}$ is $a_{m}^{a a} \lambda^{p^{\nu k}+1}$ and hence can be made unity by choice of $\lambda$ in the $G F\left[p^{2 \nu k}\right]$. For a finite field every $a$ in (18) can be made unity, so that there is a unique normal form.

17. For a general field $F$ the question is not so simple; we shall have to

* By a misprint the wrong sign is given by JoRdAN. On p. 249, 5th line from the bottom, $\lambda^{p^{\nu}} y_{m-r}^{a}$ should read $\lambda^{p^{\nu}} y_{0}^{a}$. On p. 250, 3d line from the bottom, $\lambda^{p^{\nu}}+\lambda$ should read $\lambda^{p^{\nu}}-\lambda$. 
consider normalizations not used or needed in Jordan's case. Now $S$ affects* the variables entering $(18)$ as follows :

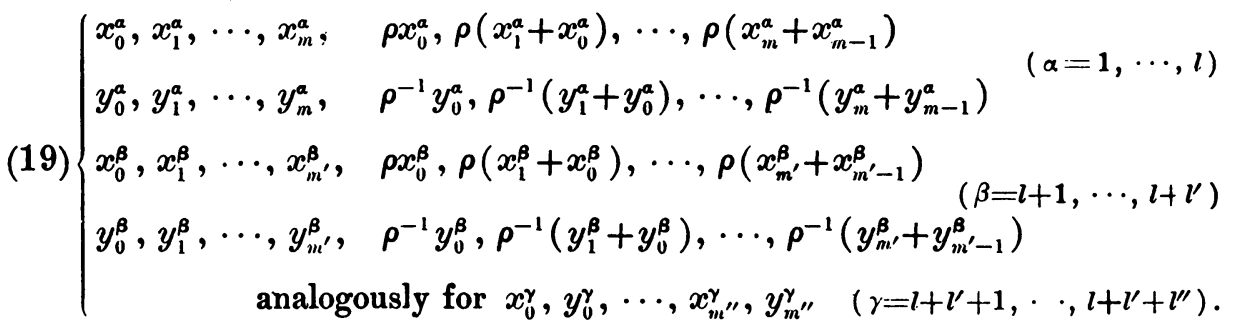

Any substitution $P$ commutative with (19) is the product of a substitution $T$ on the $x$ 's by the conjugate substitution $\bar{T}$ on the $y$ 's. Further,

$$
T=T_{m} T_{m^{\prime}} T_{m^{\prime \prime}} \ldots W,
$$

where each factor is commutative with $S, T_{m}$ denoting a substitution of the form

$$
\left|\begin{array}{ll}
x_{i}^{a} & \sum_{\delta=1}^{l} b_{a \delta} x_{i}^{\delta}
\end{array}\right| \quad(a=1, \cdots, l ; i=0,1, \cdots, m),
$$

$T_{m^{\prime}}$ being cogredient on $x_{i}^{l+1}, \cdots, x_{i}^{l+l^{\prime}}$, for $i=0,1, \ldots, m^{\prime}$, etc., while $W$ $i^{\mathrm{s}}$ derived from substitutions, the general one of which replaces $x_{i}^{a}$ by $x_{i}^{a}+\lambda x_{i-m+r}^{\beta}$ $(i=m, \cdots, m-r)$ and leaves fixed $x_{j}^{a}(j=m-r-1, \ldots, 0), r$ being $<m$. Hence $\dagger T$ replaces each $x_{0}^{a}$ by a linear function of $x_{0}^{\prime}, \ldots, x_{0}^{\prime}$ only, while

* Here and henceforth it is to be understood that the remaining variables $x\left(\rho_{1}\right), y\left(\rho_{1}\right), \cdots$, $x\left(\mu_{\nu-1}\right), y\left(\rho_{\nu-1}\right)$, conjugate to $x(\rho) \equiv x$, undergo the transformation conjugate to that on $x$. Thus the complete substitution can be expressed as a substitution on the initial variables with coefficients in $F$.

†The explicit form of $T$ is not essential to the argument; it is moreover fairly complex Linear Groups, \& 218). For $m=2, m^{\prime}=1, l=2, l^{\prime}=1, S$ is

$$
\left|\begin{array}{cc}
x_{0}^{a}, x_{1}^{a}, x_{2}^{\alpha} & \rho x_{0}^{a}, \rho\left(x_{1}^{a}+x_{0}^{a}\right), \rho\left(x_{2}^{a}+x_{1}^{\alpha}\right) \\
x_{0}^{\prime \prime \prime}, x_{1}^{\prime \prime \prime} & \rho x_{0}^{\prime \prime \prime}, \rho\left(x_{1}^{\prime \prime \prime}+x_{0}^{\prime \prime \prime}\right)
\end{array}\right|
$$

\begin{tabular}{|c|c|c|c|c|c|c|c|c|}
\hline & $x_{0}^{\prime}$ & $x_{0}^{\prime \prime}$ & $x_{1}^{\prime}$ & $x_{1}^{\prime \prime}$ & $x_{2}^{\prime}$ & $x_{2}^{\prime \prime}$ & $x_{0}^{\prime \prime \prime}$ & $x_{1}^{\prime \prime \prime}$ \\
\hline$i_{0}^{\prime}$ & $b_{11}$ & $b_{12}$ & & & & & & \\
\hline & $b_{21}$ & $b_{22}$ & & & & & & \\
\hline 1 & $c_{1}$ & $c_{2}$ & $b_{11}$ & $b_{12}$ & & & $e_{1}$ & \\
\hline "1 & $c_{3}$ & $c_{\boldsymbol{k}}$ & $b_{21}$ & $b_{22}$ & & & $e_{2}$ & \\
\hline 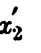 & $d_{1}$ & $d_{2}$ & $c_{1}$ & $c_{2}$ & $b_{11}$ & $b_{12}$ & $f_{1}$ & $e_{1}$ \\
\hline 2 & $d_{\mathrm{s}}$ & $d_{4}$ & $c_{3}$ & $c_{4}$ & $b_{21}$ & $b_{22}$ & $f_{2}$ & $e_{2}$ \\
\hline ונו & $g_{1}$ & $g_{2}$ & & & & & $b_{33}$ & \\
\hline "ו: & $h_{1}$ & $h_{2}$ & $g_{1}$ & $g_{2}$ & & & $k$ & $b_{83}$ \\
\hline
\end{tabular}

the explicit form of $T$ is the following (zero coefficients not heing entered) : 
$x_{m}^{\prime}, \cdots, x_{m}^{\prime}$ appear in the functions by which $T$ replaces. $x_{i}^{a}$ only when $i=m$. Analogous remarks on the $y$ 's hold for $\bar{T}$. Hence in the function by which $P \equiv T \bar{T}$ replaces $(18)$, the terms involving the variables $x_{m}^{a}$ and $y_{m}^{a}$ of maximum rank $m$ come only from the terms of (18) involving these variables. But the latter terms are given by $\phi_{a}$ of (17). Hence in order that $P$ shall transform $f_{a}^{\prime}$ given by (18) into a similar function $f_{a^{\prime}}$, it is necessary that $P$ shall transform $\phi_{a}$ into $\phi_{a^{\prime}}+\psi$, where the variables of $\psi$ are of rank $<m$. Hence the factor $T_{m}$, given by (20), of $T$ must transform $\phi_{a}$ into $\phi_{a^{\prime}}$. The necessary and sufficient conditions for this are

$$
\sum_{\alpha=1}^{\prime} a_{m}^{a a} b_{a \delta} \bar{b}_{a \epsilon}= \begin{cases}0 & \text { if } \delta \neq \epsilon, \\ a_{m}^{\prime \prime \delta \delta} & \text { if } \delta=\epsilon .\end{cases}
$$

We assume that these conditions on $T_{m}$ are satisfied. Then $T_{m}$ replaces $\sum_{a=1}^{\prime} a_{m}^{a \alpha} x_{i}^{a} y_{j}^{a}$ by

$$
\sum_{\delta, \epsilon=1}^{\prime}\left(\sum_{a=1}^{l} a_{m}^{a a} b_{a \delta} \bar{b}_{a \epsilon}\right) x_{i}^{\delta} y_{j}^{e}=\sum_{\delta=1}^{l} a_{m}^{\prime \delta \delta} x_{i}^{\delta} y_{j}^{\delta} .
$$

Hence $T_{m}$ replaces $\sum_{a=1}^{\prime} a_{m}^{a a} F_{a a m}$ by $\sum_{a=1}^{l} a_{m}^{\prime a a} F_{a a m}$. The same reasoning applies to $T_{m^{\prime}}, T_{m^{\prime \prime}}, \ldots$. Hence * if $T \bar{T}$ replaces $f_{a}^{\prime \prime}$ by $f_{a^{\prime}}^{\prime}$, the product $T_{m} \bar{T}_{m} T_{m^{\prime}} \bar{T}_{m^{\prime}} \ldots$ must replace $f_{a}$ by $f_{a^{\prime}}$.

TheOREM. Any possible transformation of $(18)$ into a similar. function by merns of a substitution commutative with $S$ can be effected by the simple substitutions (20) subject to conditions (21). The normalizution of (18) must take place in the individual sums independently.

18. In the actual normalization of $\sum_{\alpha} a_{m}^{a a} F_{a a m}$, the plan of $\$ 9$ is to apply, instead of a single $l$-ary substitution (20), a succession of binary substitutions [special cases of $(20)]$ :

$$
\left|x_{i}^{\prime} \quad x_{i}^{\prime \prime} \quad b_{11} x_{i}^{\prime}+b_{12} x_{i}^{\prime \prime}, b_{21} x_{i}^{\prime}+b_{22} x_{i}^{\prime \prime}\right| \quad(i=0,1, \cdots, m),
$$

where by $(21), a_{1 n}^{11} b_{11} \bar{b}_{12}+a_{m}^{22} b_{21} \bar{b}_{22}=0$. It follows as in $\S 9$ that $a\left(F_{11 m}+r F_{22 m}\right)$ can be multiplied by $\lambda \bar{\lambda}+r \mu \bar{\mu}$ by means of a binary substitution commutative with $S$, where $\lambda$ and $\mu$ are any rational functions of $\rho$ such that $\lambda \bar{\lambda}+r \mu \bar{\mu} \neq 0$.

19. Finally, for a root $\rho= \pm 1$, the operations take place in the initial field $F$. We may therefore follow JoRdaN's developments $\dagger$ ( $\$ \$ 33-35)$, to obtain the most general invariant $[C]$ involving the variables with multiplier \pm 1 . But the reduction of $[C]$ to normal forms by means of substitutions commuta-

* For our normalization, we may therefore dispense with the substitutions $W$. It may be be noted in passing that $W$ replaces $f_{\prime^{\prime}}$ by $f_{a^{\prime \prime}}$ only when $f_{a^{\prime}} \equiv f_{a^{\prime \prime}}$. This may be proved directly by noting that the first term in JORDAN's expression for $F_{\text {aam }}$ appears with the same coefficient after transformation by $\mathrm{W}$.

†On p. 258, 1. 7, read $2 n-2$ in the second term; at the end of $G_{a \prime \prime}$ read $x_{n-k-k^{\prime}}$. 
tive with $S$ requires essential modifications for the generalization to an arbitrary field $F$. The first step ( $(37)$ is now impossible in general. Instead,* if $F$ is any field not having modulus 2 , we employ the preliminary normalization reducing $\phi$ to $\sum_{a=1}^{l} a_{n}^{a a} x_{n}^{a} x_{n}^{a}$, each $a \neq 0$. Although $a_{n}^{11}$ is not necessarily unity, the argument in $\$ \S 38-40$ holds after an evident modification, so that we reach

$$
[C]=\sum_{a=1}^{l} a_{n}^{\alpha a} G_{a n}+\Psi \quad \text { (if } m=\text { even }=-2 n \text { ). }
$$

No changes are necessary in $§ \S 41-43$, so that there results

$$
[C]=\sum_{k=1}^{l / 2} f_{2 k-1,2 k, 2 n-1}+\Psi \quad(\text { if } m=\text { odd }=2 n-1) \text {. }
$$

Indeed, all alternate bilinear forms of determinant $\neq 0$,

$$
\sum_{i, k}^{1, \ldots} c_{i k}^{2 m} x_{i} X_{k i} \quad\left(c_{i k}=-c_{k i}, c^{\prime} \text { in field } F\right),
$$

are reducible by a linear substitution in $F$, congredient on the $x$ 's and $X$ 's, to a unique normal form $\sum_{k=1}^{m}\left(x_{2 k-1} X_{2 k}-x_{2 k} X_{2 k-1}\right)$.

Continuing similarly the reduction of $\Psi$, we obtain as a semi-canonical form of [ $C$ ] a sum of terms $G$ and $f$, affecting different variables. The factors $a$ of the $G$ are as yet any marks not zero of $F$, but otherwise the coefficients in $[C]$ are all fixed constants. As shown above for hermitian forms, so here further normalization must take place in the separate sums

$$
\sigma_{a} \equiv \sum_{a=1}^{l} a_{n}^{\alpha a} G_{a_{n}}, \quad \sum_{\beta=l+1}^{l+l^{\prime}} a_{n^{\prime}}^{\prime \beta} G_{\beta n^{\prime}}, \cdots \quad\left(n>n^{\prime}>n^{\prime \prime}, \cdots\right),
$$

the only substitution effective in normalizing $\sigma_{a}$ being of the form

$$
\mid x_{i}^{a} \quad \sum_{\delta=1}^{l} b_{a \delta} x_{i}^{\delta}: \quad(u=1, \cdots, l ; i=0,1, \cdots, n) .
$$

Now $G_{a_{n}}=x_{n}^{a} x_{n}^{a}+\sum c_{i j} x_{i}^{a} x_{j}^{a}(i \neq j)$. Further, (24) replaces $x_{i} x_{j}(i \neq j)$ by a sum of such terms. Hence if (24) replaces $\sigma_{a}$ by $\sigma_{a^{\prime}}$ it must replace $\sum a_{n}^{a a} x_{n}^{a} x_{n}^{a}$, the only terms in $\sigma_{a}$ with like subscripts to the two $x$ 's, by $\sum a_{n}^{\prime a a} x_{n}^{a} x_{n}^{a}$, the only terms in $\sigma_{a^{\prime}}$ with like subscripts to the two $x$ 's. Hence, since $F$ does not have modulus 2, we derive the necessary conditions

$$
\sum_{a=1}^{l} a_{n}^{a a} b_{a \delta}^{2}=a_{n}^{\prime \delta \delta}, \quad \sum_{a=1}^{\prime} a_{n}^{a a} b_{a \delta} b_{a \varepsilon}=0(\delta, \varepsilon=1, \cdots, l ; \delta \neq \varepsilon) .
$$

* Some corrections make the reading of $\xi \xi 37-39$ easier. In $z \xi 37,38$, read $[C]$ for $[a a]$. In $\xi 38$ the use of $a_{2 n}^{11}$ instead of $a_{n}^{11}$ does not conform to the earlier notations. For $T$ in $z 39$ read

$$
T=\left|x_{2 n}^{\prime}, \cdots, x_{2 n-2 k}^{\prime} \quad x_{2 n}^{\prime}+\lambda x_{2 k}^{\prime}, \cdots, x_{2 n-2 k}^{\prime}+\lambda x_{0}^{\prime}\right| \text {. }
$$


But these are also sufficient conditions that (24) shall replace $\sigma_{a}$ by $\sigma_{a^{\prime}}$. Indeed, if (25) hold, (24) replaces $\sum_{a} a_{n}^{a a} x_{i}^{a} x_{j}^{a}(i \neq j)$ by

$$
\sum_{\delta, \epsilon}^{1, \ldots, l}\left(\sum_{a=1}^{l} a_{n}^{a a} b_{a \delta} b_{a \epsilon}\right) x_{i}^{\delta} x_{j}^{\epsilon}=\sum_{\delta=1}^{l} a_{n}^{, \delta \delta} x_{i}^{\delta} x_{j}^{\delta} .
$$

Hence for the purposes of normalization, $G_{a n}$ of $\sigma_{a}$ may be replaced by its lexding term $x_{n}^{a} x_{n}^{a}$ and the subscript $i$ in (24) restricted to the value $n$. The problem is therefore essentially the normalization of an l-ary quadratic form within the group of all l-ary substitutions in the field $F$.

20. With regard to fields having modulus 2 , we restrict our attention to a finite field, say the $G F\left[2^{k}\right]$. Then every mark is a square. The developments of JoRDAN ( $\S 46-53$ ) hold for the $G F\left[2^{k}\right]$ after slight changes. In $\S 46$ we must multiply ${x_{n}^{\prime 2}}^{2}+x_{n}^{\prime \prime 2}$ by $\lambda$, where $\lambda$ is any fixed mark such that $\lambda x^{2}+\lambda y^{2}+x y$ is irreducible in the $G F\left[2^{n}\right]$ (cf. Linear Groups, $\S 199$ ). Hence in $\S 47, G_{1 n}+G_{2 n}$ must be multiplied by $\lambda$; similarly in the latter sections. In $\S 48$ the case $b^{a} \neq 0$ can be reduced to the case $b^{a}=1$ by applying the substitution which multiplies $x_{i}^{a}$ by $\left(b^{a}\right)^{-\frac{1}{2}}$ for $i=0,1, \ldots, m$.

The University of Chicago, November 9, 1905. 